\title{
Risk Adapted Strategies in the Management of Thyroid Cancer
}

\author{
Jatin P Shah, MD* and Cristina Valero Mayor*, MD \\ From: "The Head and Neck Service, Memorial Sloan Kettering Cancer Center \\ New York, NY. 10065, and the Dept. of Oncology and Reconstructive Surgery, \\ Sechenov University, Moscow, Russia
}

\section{Summary:}

There is a rising incidence of thyroid cancer world wide in the past four decades. Such a rise is observed most often in patients between the ages of 50-65. However, a majority of these newly discovered cancers are small, less than $2 \mathrm{~cm}$ in size, intrathyroidal papillary carcinomas. Understanding the tumor progression model of follicular cell derived cancers and appreciating the impact of prognostic factors allows the development of risk group stratification at initial diagnosis and after surgical treatment. Employing risk group stratification permits discretion in selection of surgical treatment, in the use of adjuvant therapies, and the subsequent follow up strategies. Molecular analysis of aggressive cancers opens a path to the use of targeted therapies in patients with iodine resistant recurrent or metastatic thyroid cancers. A great majority of thyroid cancer patients fall into the low and intermediate risk groups, and their long-term outcomes remain excellent. Therefore, in conclusion, understanding the biology of thyroid cancer and appreciation of prognostic factors and employing risk group stratification is crucial for cost effective treatment of cancer of the thyroid gland.
The incidence of thyroid cancer is rising in the United States significantly, with a nearly 8 -fold increase in the past four decades. ${ }^{1}$ The majority of these newly diagnosed patients are women, although there is a modest rise in men also. However, an important fact to notice is that in spite of a steep rise in the incidence of thyroid cancer, the cancer-related mortality remains the same. This may be due to the fact that the majority of the newly diagnosed cancers are non-fatal cancers. On the other hand, one can conclude that very little progress is made in reducing mortality from thyroid cancer. A similar rise in the incidence of thyroid cancer is seen worldwide. ${ }^{2-4}$ Important observations can be made from the global data, showing that the majority of newly diagnosed thyroid cancers are not in young people, but in older people between the age of 55 and 64. Majority of these are localized cancers relatively confined to the thyroid gland without dissemination to the regional lymph nodes or to distant sites, and the majority of these are papillary carcinomas. ${ }^{2}$ Another study from the United States reported that majority of the newly diagnosed cancers are small, subcentimeter carcinomas (less than $1 \mathrm{~cm}$ ). ${ }^{5}$ Many of these cancers are incidentally discovered on imaging studies such as Computerized Tomography (CT), Magnetic Resonance Imaging (MRI) or Ultrasound (US) done for other purposes. One of the reasons for the steep rise in thyroid surgery globally, is the discovery of these small subclinical cancers. 


\section{Pathology and Biology}

In order to deliver cost effective care to our patients, we need to understand the pathology and biology of these follicular cell derived thyroid cancers (Figure 1). A cancer developing in thyroid follicular cell may differentiate into a papillary carcinoma, follicular carcinoma, or most often a mixed papillary and follicular cancer. This type of tumor heterogeneity is quite common in differentiated thyroid carcinoma. Nearly $80-85 \%$ of patients with thyroid cancer present with a papillary or follicular carcinoma with a good prognosis. About $10 \%$ go further along this tumor progression model and present with an insular or tall cell variant of papillary carcinoma. A smaller number of patients go further along this tumor progression model and present with a poorly differentiated carcinoma and these are serious cancers with a significantly adverse impact on prognosis. Rarely these tumors go on further to progress to anaplastic carcinoma $(<1 \%)$. Anaplastic carcinomas carry a grim prog- nosis with a median survival time of less than two years in most patients. Indirect evidence supporting this tumor progression model is seen with tumor heterogeneity in patients with poorly differentiated carcinoma, where, areas of well differentiated papillary carcinoma and areas of poorly differentiated carcinoma are seen within the same tumor, implying that the tumor started off as a differentiated cancer and progressed to a poorly differentiated carcinoma. The other indirect evidence is shown in patients who develop multiple recurrences of papillary carcinoma. With each recurrence there is progressive anaplasia eventually leading to poorly differentiated or anaplastic transformation. As this tumor progression takes place histologically, there are clinical manifestations of the tumor, which would lead one to the diagnosis of a thyroid cancer with worsening prognosis. As this tumor progression occurs, tumor size starts increasing. As the tumor gets bigger the risk of extra thyroid extension increases and the risk of distant metastases also increases with a rise in mortality.

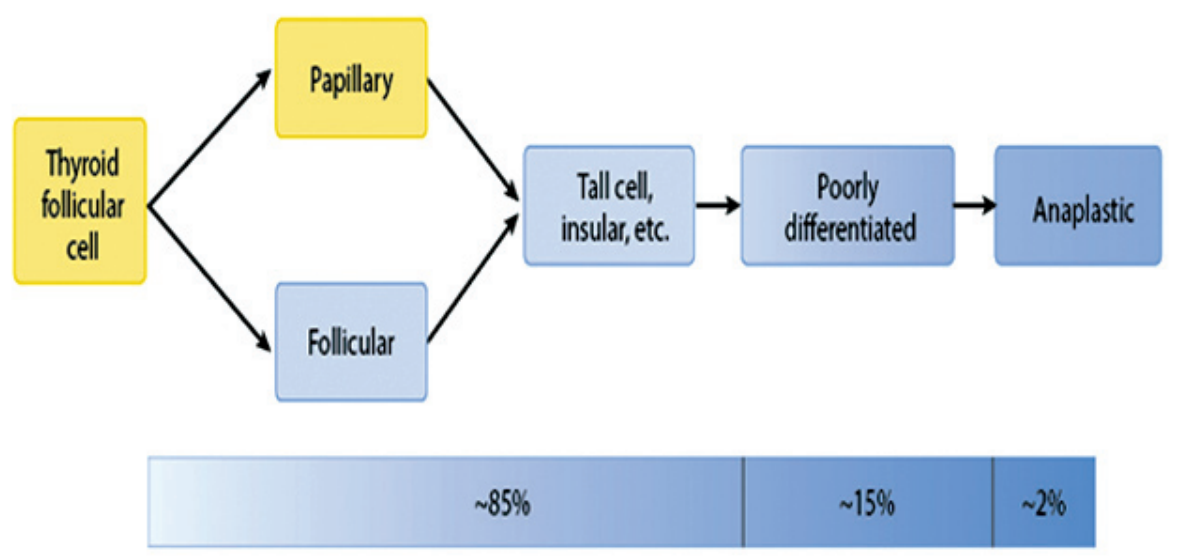

Fig.1. Tumor progression of thyroid cancer from differentiated to anaplastic carcinoma (From Jatin Shah's Head and Neck Surgery and Oncology - 5th edition, Elsevier, 2019)

\section{Prognostic Systems (AJCC Staging and ATA Risk groups)}

In order to provide optimal treatment to the patient we need to have clinical as well as pathological parameters of the cancer to assess prognosis. There are two prognostic systems available which assess the risk of death and the risk of recurrence. The American Joint Committee on Cancer (AJCC) stating system is devised for estimation of the risk of death due to cancer. The current $8^{\text {th }}$ edition of the AJCC staging system has introduced three major changes. ${ }^{6}$ The age for risk stratification is now 55. In addition, there are changes in $\mathrm{T}$ staging as well as $\mathrm{N}$ staging. Microscopic extrathyroid extension does not upstage the T stage, and all lymph nodes in the central compartment (Level VI and VII) are now staged as N1a, and lymph nodes in the lateral neck remain as N1b. These changes have significantly down staged many patients who would have been staged higher in the past due to the age cutoff at 45 and due to upstaging $\mathrm{T}$ status because of presence of microscopic extrathyroid extension. These changes in the $8^{\text {th }}$ edition of the AJCC staging system have shown comparable survival rates at 10 years for age cutoff at 45 and 55. Patients under age 55 can only be Stage I or Stage II. On the other hand, patients older than 55 can be staged as stages I, II, III or IV, with increasing mortality with progression of stage. 
The American Thyroid Association (ATA) also has a risk group stratification system, which in contrast to survival, identifies patients at a low, intermediate or high risk for tumor recurrence. ${ }^{7}$ This risk group stratification system uses similar parameters as in the AJCC staging system, but the stratification is focused on recurrence rather than death.

\section{Risk Group Stratification}

The prognostic factors used by both, AJCC and ATA have been identified as independent factors by multivariable analyses in several different datasets. The earliest amongst these are the reports from Mayo and Lahey clinics identifying patient's age, histo- logical grade, extrathyroid extension, tumor size, completeness of resection, and distant metastases as independent prognostic factors. ${ }^{8-10}$ Analyses of the data from Memorial Sloan Kettering Cancer Center confirms a strong impact of these factors on long-term outcomes. Patient's age, sex, histology and size of the primary tumor, as well as gross extrathyroid extension show a significant impact on outcome (Figure 2). Lymph node metastasis in general does impact on outcome with long-term decline in survivorship. However, nodal metastasis has no impact on survivorship in patients under 55, the negative impact of nodal metastasis occurs only in the older age group (Figure 3).
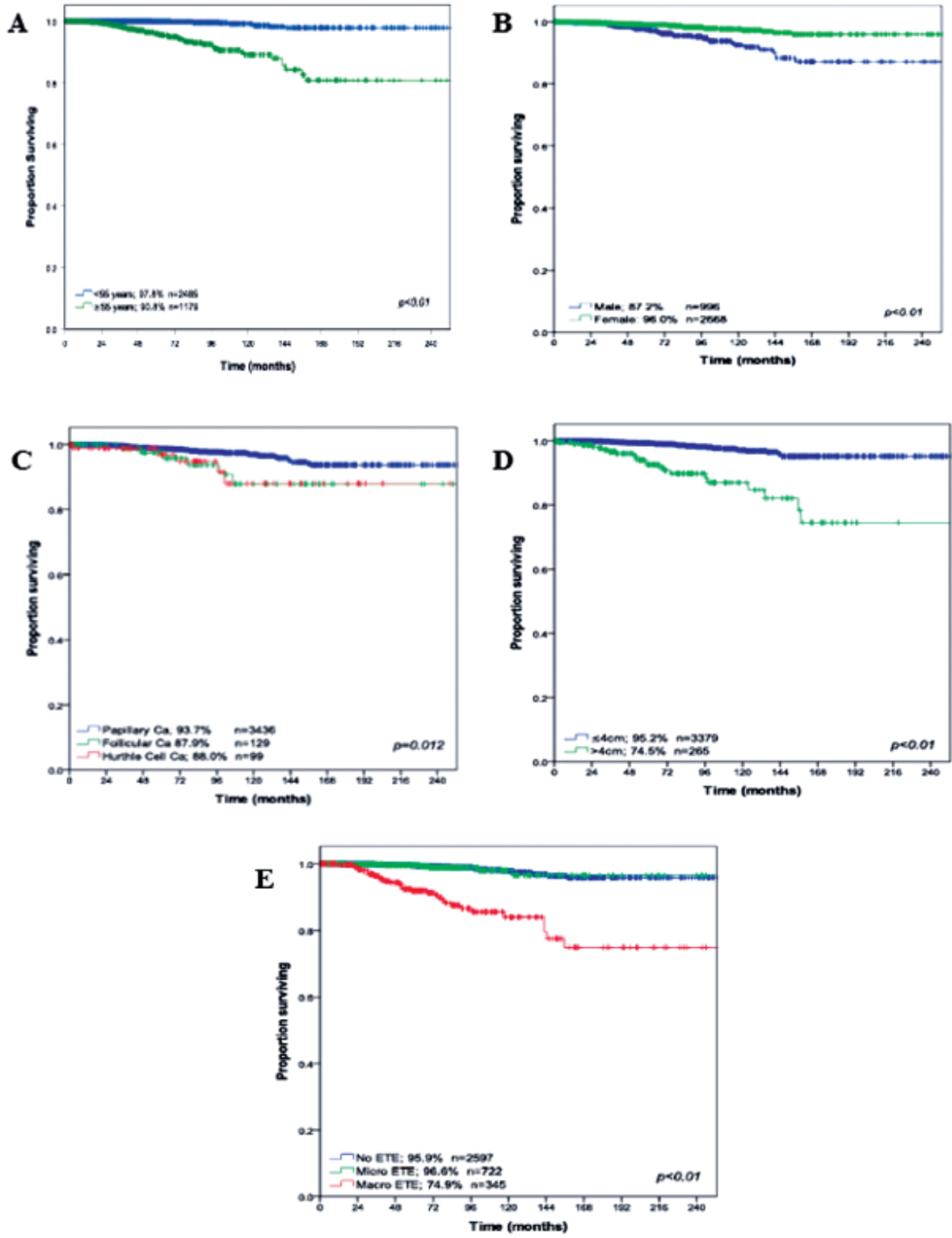

Ca., Carcinoma; Micro, microscopic; ETE, extra thyroid extension; Macro, macroscopic

Fig. 2. Disease-specific survival according to (A) age, (B) sex, (C) histology, (D) size of the primary tumor, and $(E)$ extra thyroid extension (from Jatin Shah's Head and Neck Surgery and Oncology $-5^{\text {th }}$ edition, Elsevier, 2019) 

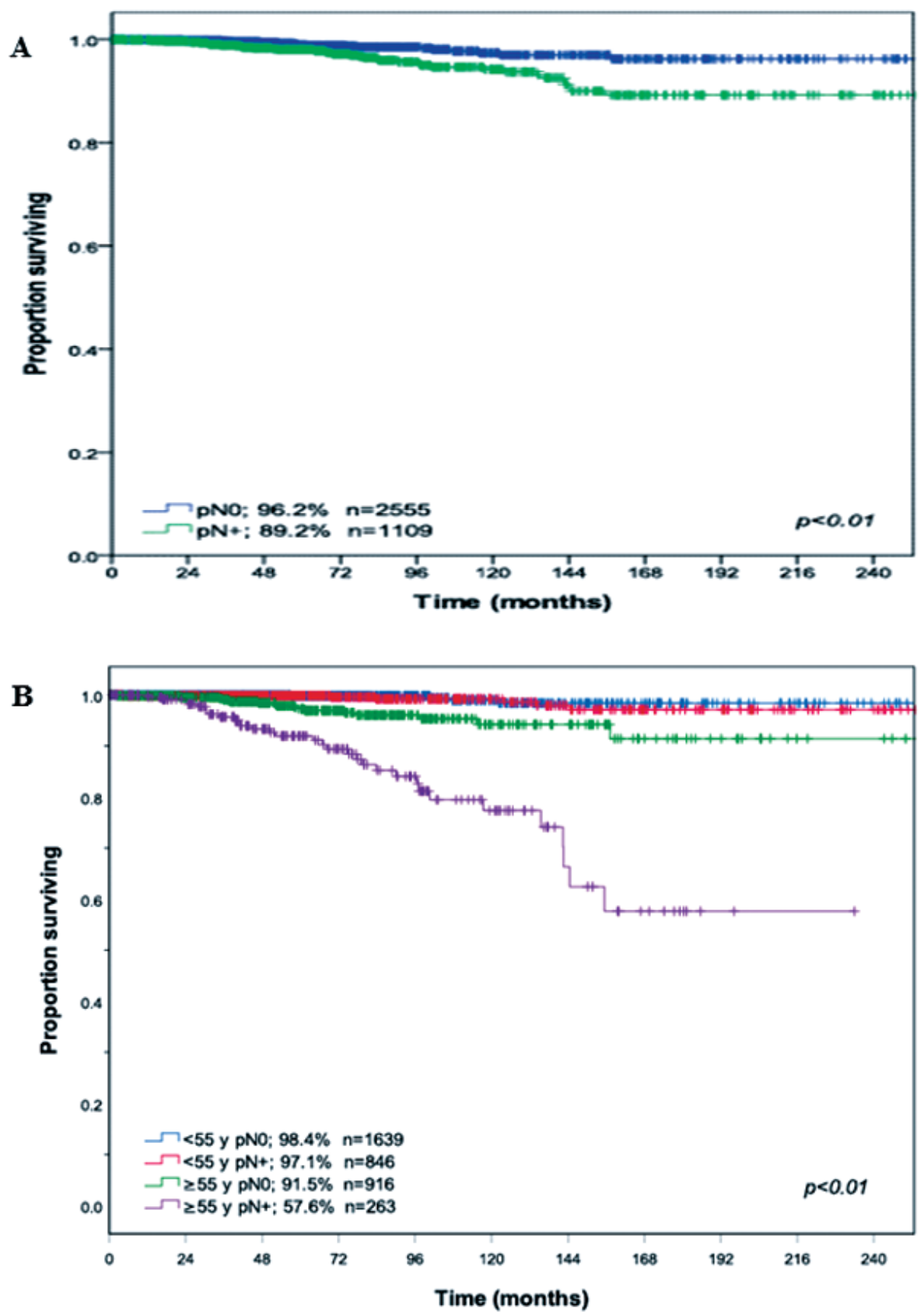

$\mathrm{pN} 0$, Negative nodal classification; $\mathrm{pN}+$, positive nodal classification

Fig. 3. Disease-specific survival according to nodal status in all patients (A) and stratified by age by age (B) (From Jatin Shah's Head and Neck Surgery and Oncology - 5th edition, Elsevier, 2019)

Combining these prognostic factors allows us to stratify patients into risk groups (Figure 4). Low risk patients are young patients, generally females, with tumors less than $4 \mathrm{~cm}$ in diameter, without extrathyroid extension, well differentiated histology and no distant metastasis. On the other hand, high risk group comprises of older patients, generally men, with tumors larger than $4 \mathrm{~cm}$, or with gross extrathyroid extension, poorly differentiated histology, or presence of distant metastasis. These are the two well defined groups, low risk and high risk. But there is an intermediate risk group, which consists of older patients with low risk cancers, and younger patients with high risk cancers. The 20 year survival according to the aforementioned risk groups is shown in Figure 5. Disease-specific survival at 20 years in the low risk group was $100 \%$, while in the high risk group it dropped to $77 \%$, and in the intermediate risk group it was 9697\%. Employing risk group stratification in selec- 


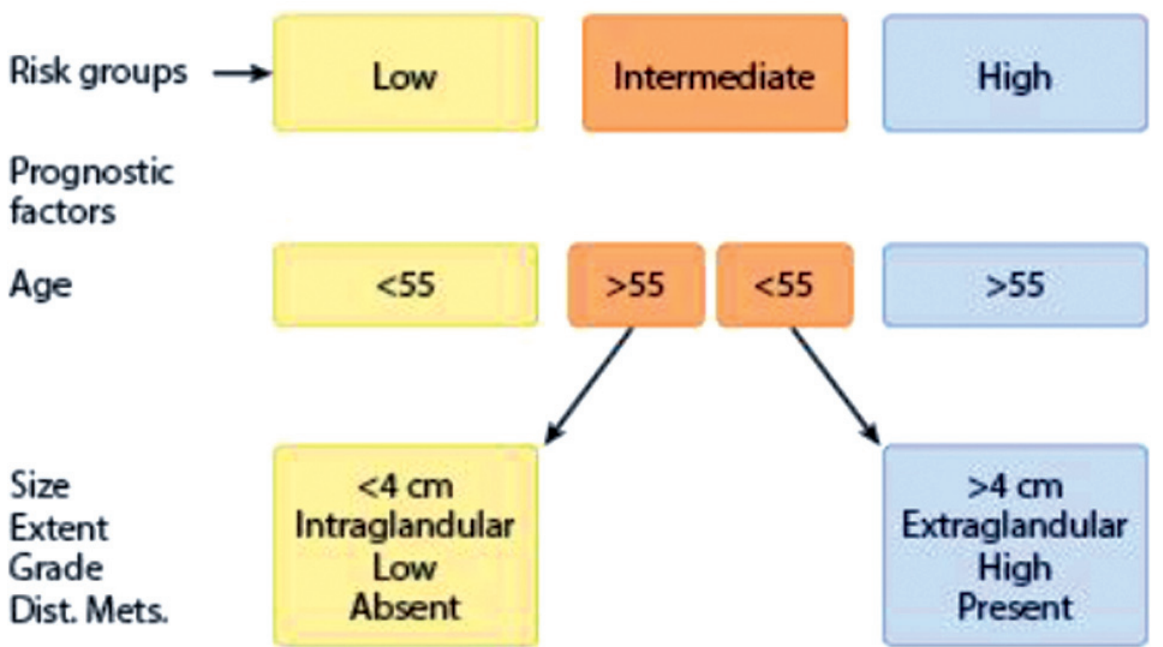

Fig. 4. Risk group categories (From Jatin Shah's Head and Neck Surgery and Oncology $5^{\text {th }}$ edition, Elsevier, 2019)

Dist. Mets, Distant metastases

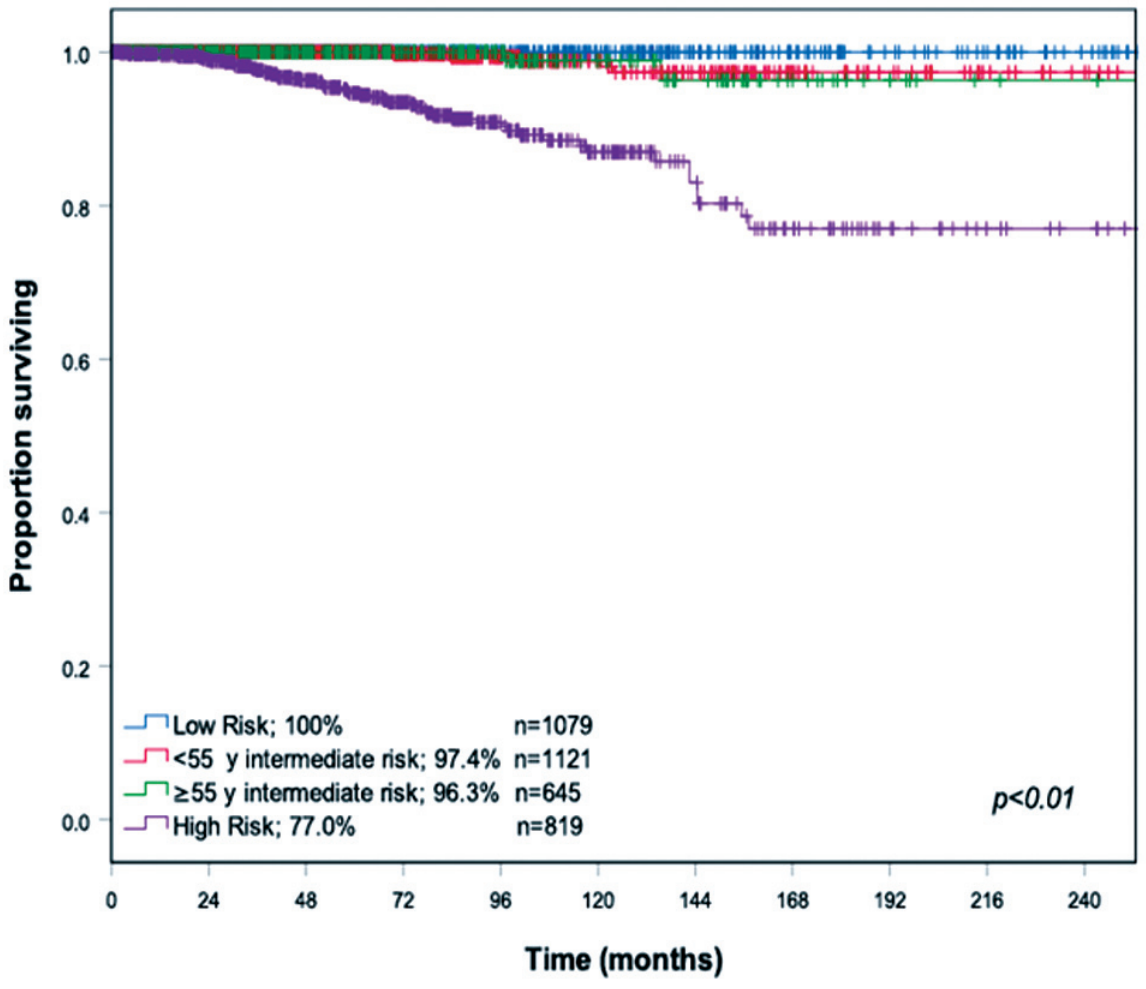

Fig. 5. Long-term disease-specific survival in relation to risk-group stratification (From Jatin Shah's Head and Neck Surgery and Oncology - 5th edition, Elsevier, 2019)

tion of treatment offers optimal and cost effective treatment to each patient and minimizes sequela of treatment in the low risk group.

According to the ATA stratification, low risk patients are patients under 55 and those with intrathyroidal tumors and/or those with very low volume lymph node involvement. This means less than 5 metastatic lymph nodes or less than $2 \mathrm{~mm}$ deposit of microscopic focus in the lymph node. In the low risk patients there is no indication for adjuvant therapy. Intermediate risk group patients are those with poorly differentiated histology, minor extrathyroid extension, extensive vascular invasion or more than 5 positive lymph nodes. And high risk group patients are those with gross extrathyroid extension, incomplete resection, presence of distant metastasis or lymph nodes greater than $3 \mathrm{~cm}$. These patients have a higher risk of recurrence.

In comparing AJCC staging system and ATA risk groups, one notices that for patients under 55 in AJCC staging there are only two stages. There is no Stage III or Stage IV, and similarly in the ATA 
risk categories the highest risk patients are only those with gross extrathyroid extension or distant metastasis. Thus, the majority of patients in AJCC stage I, would fall into the ATA low risk or intermediate risk category. These patients have low risk of death and low risk of recurrence. It is only the older patients who have the disease in Stage III or Stage IV, who fall into the high risk category of ATA for recurrence. In an analysis of nearly 5000 patients treated at MSKCC, only $6 \%$ were in the ATA high risk category and $2 \%$ with higher AJCC stage at the time of treatment. ${ }^{11}$ Thus, on an average, about $5 \%$ of patients need aggressive treatment, including aggressive surgery, aggressive adjuvant treatment and intensive post treatment follow-up.

\section{Treatment - Surgery for the primary tumor}

Risk group stratification at initial diagnosis for selection of surgical treatment, and postsurgery for assessing the need for adjuvant therapy is critical for delivering cost effective optimal treatment for thyroid cancer. For patients with unifocal or multifocal intrathyroidal primary tumor confined to one lobe, ipsilateral lobectomy is curative, if the opposite lobe is grossly normal. It is important to emphasize however, that all operations done for thyroid cancer must be "extracapsular", leaving no thyroid tissue at the site of surgery. The operations called subtotal thyroidectomy and near total thyroidectomy, where some thyroid tissue is left behind, requiring "remnant ablation" with radioactive iodine, are not cancer operations, and should not be performed. Thus, there are only two operations for thyroid cancer; lobectomy or totally thyroidectomy, both of which are extracapsular. There should be no residual thyroid tissue left behind at the site of the operation.

Tumor control and survival are comparable in patients with intrathyroidal, unifocal or multifocal tumors confined to one lobe undergoing lobectomy or total thyroidectomy. In a study of over 800 patients, comparing of lobectomy vs total thyroidectomy the local recurrence, regional recurrence, distant recurrence, recurrence free survival, overall survival and disease-specific survival were very similar showing no statistical difference in any of the outcome measures. ${ }^{12}$ In another study from the National Cancer Data Base (NCDB) of nearly 62,000 patients, no long-term difference in diseasespecific survival was seen between lobectomy and total thyroidectomy. ${ }^{13}$
On the other hand, total thyroidectomy is indicated in patients with bilateral disease, bilateral gross nodules, gross extra thyroid extension, poorly differentiated histology in a large tumor, extensive lymph node metastases, or in patients with distant metastases. In addition, patients who present with gross extrathyroid extension, involving other structures, such as strap muscles, trachea, larynx, esophagus or recurrent laryngeal nerve, need more extensive operatrions in addition to total thyroidectomy. The principle of surgery for such extensive tumors is to achieve an R0 resection, removing all gross tumor. Every attempt should be made to preserve functioning structures and vital structures, maintaining a balance between tumor control and best functional outcome. Although adjuvant treatment with radioactive iodine or external radiation therapy is available, it does not compensate for inadequate or incomplete surgery. Achieving a R0 resection clearly offers a significant benefit in survival compared to patients who had R2 resection. ${ }^{14}$

\section{Cervical Lymph Nodes}

Regional spread to cervical lymph nodes with micro metastases is quite common and occurs in nearly $50 \%$ of patients with papillary carcinoma. However, micro metastases have no prognostic significance in low risk patients, and they do not impact upon outcome. Thus, elective lymph node dissection is not recommended in low risk patients. However, elective regional node dissection, particularly of the central compartment lymph nodes may be considered in older patients, with large primary tumors with gross extrathyroid extension or poorly differentiated histology. Therapeutic dissection is indicated for metastatic lymph nodes palpated clinically, seen on imaging studies such as US or CT, and if gross metastases are discovered during thyroidectomy. When a regional lymph node dissection is performed it should be compartmental and comprehensive. Removal of only few enlarged lymph nodes ("Berry picking") is not recommended, since it leads to regional recurrence in as many as $30 \%$ of patients. However, if initial node dissection is complete, then the regional recurrence is only $3 \%$. Comprehensive clearance of lateral neck lymph nodes requires removal of lymph nodes from levels II-V and central compartment node dissection requires removal of all lymph nodes from levels VI and VII. The patterns of neck metastasis from thyroid cancer 
are well known. The first echelon lymph nodes are confined to the central compartment $(\mathrm{N} 1 \mathrm{a})$, or levels VI and VII. Subsequent spread occurs to the lateral neck (N1b), which includes levels II, III, IV and V. Metastatic disease at level I is rarely seen, and it is usually present when there is extensive nodal disease in the lower levels of the neck.

\section{Adjuvant Therapy}

Adjuvant therapy with Radioactive iodine (RAI) is indicated in patients with residual disease at the primary site, extensive nodal metastases, and those with distant metastases. Thyroglobulin (TGb) is a reliable biomarker for differentiated thyroid cancer, and patients with persistently elevated $\mathrm{TGb}$, after surgery are also candidates for RAI therapy. Routine use of RAI, for remnant ablation is not required, if the surgical procedure is a total extracapsular thyroidectomy and the patient has no measurable TGb six weeks following surgery. Unindicated routine use of RAI, is not beneficial to the patient and can even be harmful leaving long term sequela of RAI, with xerostomia, loss of taste and recurrent episodes of sialadenitis in some patients. Furthermore, with increasing doses of RAI, there is a significant risk of a second primary cancer. ${ }^{15-18}$ It is important to remember that RAI has no effect on patients with poorly differentiated thyroid carcinoma, where the tumor has lost iodine avidity. In such patients, with residual or recurrent disease, if $\mathrm{TGb}$ is not elevated, then RAI is not recommended. Such patients would be candidates for external beam radiotherapy (RT), with the expectation of local control of disease.

\section{Distant metastasis}

The patients who present with distant metastases, or who develop distant metastases later on are in need for systemic therapy. However, if the distant metastases produce elevated levels of $\mathrm{TGb}$, or are demonstrable on a RAI tracer scan, then the first mode of treatment is with RAI. For proper and effective dose of RAI, a dosimetry is recommended. However, for RAI to be effective, the metastatic lesions have to be still well differentiated carcinoma, which show iodine avidity. As we have previously mentioned, RAI does not work in poorly differentiated thyroid carcinoma. However, patients with tumor heterogeneity can have some metastatic lesions that are well differentiated, and thus iodine avid, while there may be other lesions which are poorly differentiated, and thus not avid to RAI. This is often demonstrated, on imaging studies, where the lesions seen on a Positron Emission Tomography (PET) scan for example, are not seen on a RAI tracer scan and those lesions seen on a RAI tracer scan are not FDG (fluoro deoxy glucose) avid on a PET scan due to their low level of metabolic activity. RAI in such patients will work only on the well differentiated metastatic lesions, while the more aggressive poorly differentiated lesions will not benefit from RAI.

In the immediate post operative period after total thyroidectomy, the first step would be measurement of thyroglobulin at 6 weeks following surgery. If the thyroglobulin is elevated, then that patient should have appropriate imaging studies, including a RAI tracer scan as well as a PET scan to localize the metastatic lesions. Treatment with RAI is appropriate in this setting. If the thyroglobulin is not elevated but the patient has extensive local disease, particularly poorly differentiated carcinoma, then that patient should get external beam radiation therapy for local control of disease in the neck. Patients with extensive poorly differentiated carcinoma, may sometimes have elevated TGb, and such patients are candidates for both, external RT and RAI treatment. Some patients with metastatic lesions in critical locations may even need surgery for metastasis, for example bone metastasis in a weight bearing bone, or in the spine, with cord compression.

Cytotoxic chemotherapy has proven to be disappointing in the treatment of metastatic thyroid cancer. However, targeted therapies are playing increasing role in the treatment of metastatic thyroid cancer. Whenever feasible, tumors should be studied for mutations such as BRAF V600E, RET, RAS and TERT. BRAF inhibitors are currently employed either in clinical trials or on a compassionate basis. ${ }^{19}$ Thus, in patients with systemic disease, whenever available, genome sequencing of the tumor should be performed to identify actionable targets, and then one would be able to choose the appropriate drug with the expectation of tumor response, prolongation of life and improved quality of life.

\section{TSH Suppression}

All patients with thyroid cancer should have their thyroid stimulating hormone (TSH) suppressed to prevent development of recurrent or metastatic disease from TSH dependent differentiated thyroid 
cancer. However, excessive suppression of TSH is not desirable, since it leads to increasing risk of osteoporosis, with its detrimental effect on the patient. Thus, the level of suppression to reduce the risk of recurrence should be balanced against the harmful effect of excessive suppression. TSH levels between 0.5 and $1 \mathrm{mU} / \mathrm{L}$, are considered optimum to reduce the risk of recurrence and minimize the incidence of osteoporosis.

\section{Follow up and Surveillance}

The intensity and frequency of follow up visits arealso related to the risk groups. Young patients with a low risk tumor may be seen every six months for the first two years, and thereafter annually. In general patients with low risk small primary differentiated carcinomas, require clinical examination and blood studies for TGb, T4 and TSH during the follow up visit. Routine ultrasound (US) examination in all patients at each follow up visit is not recommended. The practice of routine US examination at each visit will sometimes identify small subcentimeter lymph nodes in the central compartment, leading to a fine needle aspiration cytology (FNAC) and surgical intervention, which may have no impact on long-term outcome. Follow up US examination is warranted in patients with high risk of recurrence, such as those with large primary tumors with extrathyroid extension, or multiple nodal metastases at initial surgery. In such patients, even anatomic imaging such as CT scan, MRI or a PET scan may be necessary. We generally do not recommend an FNAC on subcentimeter lymph nodes seen on an US. Such lymph nodes are followed with a repeat US in six months, and if growth is observed, then an FNAC is recommended. Similarly, a routine annual RAI tracer scan is not recommended for follow up. Instead, follow up with $\mathrm{TGb}$ is accurate in patients with differentiated carcinoma, and anatomic imaging for structural disease is recommended in patients with poorly differentiated carcinoma.

\section{References}

1. Lim H, Devesa SS, Sosa JA, Check D, Kitahara CM. Trends in thyroid cancer incidence and mortality in the United States, 1974-2013. JAMA. 2017;317(13):1338-1348.

2. Pellegriti G, Frasca F, Regalbuto C, Squatrito S, Vigneri R. Worldwide increasing incidence of thyroid cancer: update on epidemiology and risk factors. J Cancer Epidemiol. 2013;2013:965212.

3. Vaccarella S, Franceschi S, Bray F, Wild CP, Plummer M, Dal Maso L. Worldwide Thyroid-Cancer Epidemic? The Increasing Impact of Overdiagnosis. N Engl J Med. 2016 Aug 18;375(7):614-7.

4. Maniakas A, Davies L, Zafereo ME. Thyroid Disease Around the World. Otolaryngol Clin North Am. 2018 Jun;51(3):631-642.

5. Davies L, Welch HG. Current thyroid cancer trends in the United States. JAMA Otolaryngol Head Neck Surg. 2014 Apr;140(4):317-22.

6. Amin MB, Edge SB, Greene F, et al (eds). American Joint Committee on Cancer. AJCC Cancer Staging Manual, Eighth Edition. Geneva, Switzerland: Springer; 2017.

7. American Thyroid Association. About the ATA. Available at: https://www.thyroid.org/about-american-thyroid-association (accessed October 4, 2019).

8. Hay ID, Grant CS, Taylor WF, McConahey WM. Ipsilateral lobectomy versus bilateral lobar resection in papillary thyroid carcinoma: a retrospective analysis of surgical outcome using a novel prognostic scoring system. Surgery. 1987 Dec;102(6):1088-95.

9. Cady B, Rossi R. An expanded view of risk-group definition in differentiated thyroid carcinoma. Surgery. 1988 Dec;104(6):947-53.

10. Hay ID, Bergstralh EJ, Goellner JR, Ebersold JR, Grant CS. Predicting outcome in papillary thyroid carcinoma: development of a reliable prognostic scoring system in a cohort of 1779 patients surgically treated at one institution during 1940 through 1989. Surgery. 1993 Dec;114(6):1050-7; discussion 1057-8.

11. Ghaznavi SA, Ganly I, Shaha AR, English C, Wills J, Tuttle RM. Using the American Thyroid Association Risk-Stratification System to Refine and Individualize the American Joint Committee on Cancer Eighth Edition Disease-Specific Survival Estimates in Differentiated Thyroid Cancer. Thyroid. 2018 Oct;28(10):1293-1300.

12. Harries V, Wang LY, McGill M, et al. Should multifocality be an indication for completion thyroidectomy in papillary thyroid carcinoma? Surgery. 2019 Sep 9. pii: S0039-6060(19)30512-4.

13. Adam MA, Pura J, Gu L, et al. Extent of surgery for papillary thyroid cancer is not associated with survival: an analysis of 61,775 patients. Ann Surg. 2014 Oct;260(4):601-5; discussion 605-7.

14. Wang LY, Nixon IJ, Patel SG, et al. Operative management of locally advanced, differentiated thyroid cancer. Surgery. 2016 Sep;160(3):73846.

15. Sawka AM, Thabane L, Parlea L, et al. Second primary malignancy risk after radioactive iodine treatment for thyroid cancer: a systematic review and meta-analysis. Thyroid. 2009 May;19(5):451-7.

16. Iyer NG, Morris LG, Tuttle RM, Shaha AR, Ganly I. Rising incidence of second cancers in patients with low-risk (T1N0) thyroid cancer who receive radioactive iodine therapy. Cancer. 2011 Oct 1;117(19):4439-46.

17. Marti JL, Jain KS, Morris LG. Increased risk of second primary malignancy in pediatric and young adult patients treated with radioactive iodine for differentiated thyroid cancer. Thyroid. 2015 Jun;25(6):681-7.

18. Khang AR, Cho SW, Choi HS, et al. The risk of second primary malignancy is increased in differentiated thyroid cancer patients with a cumulative (131)I dose over 37 GBq. Clin Endocrinol (Oxf). 2015 Jul;83(1):117-23.

19. Crispo F, Notarangelo T, Pietrafesa M et al. BRAF Inhibitors in Thyroid Cancer: Clinical Impact, Mechanisms of Resistance and Future Perspectives. Cancers (Basel). 2019 Sep 18;11(9). pii: E1388. 